\title{
I'll Have What She's Having: Gauging the Impact of Product Placements on Viewers
}

\author{
Sharmistha Law, University of Toronto \\ Kathryn A. Braun, Harvard Business School
}

Product placement in TV shows is becoming increasingly common, yet little is known about its effectiveness nor even how to define and measure such effectiveness. This research examined the effectiveness of product placement with the use of two different types of measures: explicit measures that tap memory directly (with the use of a recognition and recall task), and an implicit measure that measures the effect of exposure on product choice indirectly. It was hypothesized that the ability of product placement to enhance memory and choice may be mediated by distinct mechanisms. The results showed an overall enhancement in product recall, recognition, and choice due to placement. Further, although products central to the plot were remembered and recalled more than products placed more subtly, no reliable effect of centrality of placement was observed on the choice measure. This dissociation also occurred with modality of placement-where seen-only products most influenced choice but were least recalled. The present data conform to theories of memory, which predict that performance on explicit memory task can be dissociated or uncorrelated with performance on implicit performance or choice. This finding has significant implications for how product placements are designed and how their efficacy evaluated.

Cosmo Kramer says he craves Kenny Roger's roasted chicken in an episode of Seinfeld. Jimmy Smit's character on NYPD Blue chooses Snapple to quench his thirst. And Helen Hunt of Mad About You reaches for Tums to cure her tummy ache. Product placements, the ultimate in the subtle sell, were once the sole domain of movies but are now making their way to the small screen. Placements are now seen as an economically viable alternative to network advertising: A company can pay $\$ 200,000$ for a season's worth of placements or $\$ 475,000$ for a single 30 s commercial within the same type of primetime programming (Wells, 1996). Placements are viewed as a way for manufacturers to create familiarity, and in an indirect way, celebrity endorsements, for their product (Buss, 1998). 
Are product placements effective? The most common measure of product placement effectiveness has been consumer memory, and the evidence is inconclusive. There has been some support for the notion that product placements facilitate brand memory (Babin \& Carder, in press; Vollmers \& Mizerski, 1994). Babin and Carder (in press) for instance, had one group of participants view a film containing brand placements. Later, participants were given product category names as cues and asked if they recalled any brand names from the recently viewed film. Compared to a control group, who did not view the film, approximately $25 \%$ of the placed brands were better remembered by the participants in the exposure condition. Other investigators, in contrast, have reported inconsistent or insignificant effects of placement on brand memory (Babin \& Carder, 1996; Karrh, 1994). In summary, although product placements are becoming prevalent, there is little knowledge about whether they are effective and how to best measure their impact.

One of the key features of previous studies of product placement is that they have employed explicit tests of memory that make direct reference to the prior exposure. Specifically, the impact of product placement has typically been measured by free recall, where participants are asked to recall all brands seen in the movie just viewed (e.g., d’Astous \& Chartier, 1999; Gupta \& Lord, 1998; Vollmers \& Mizerski, 1994); aided recall, where people are given a product category cue and then asked to recall (e.g., Babin \& Carder, in press; Karrh, 1994); and recognition, where they are asked to judge whether they remember seeing a brand in the movie (e.g., Babin \& Carder, 1996; Gupta \& Lord, 1998). These tests are called explicit (or direct) measures of memory because participants are directly tested on episodes from their recent past.

But it is now known that past experience can affect us in many ways, not all of which may be apparent to introspection. Indeed, there is growing evidence that consumer decisions are often influenced by a sense of familiarity or without the recollection of the study episode. For example, the truth effect (the increased belief in an ad claim on account of a previous exposure, Hawkins \& Hoch, 1992; Law, Hawkins, \& Craik, 1998), the mere exposure effect (the formulation of a positive affect from exposure to a brief stimulus, Janiszewski, 1993), and the false familiarity effect (mistakenly judging a hypothetical brand to be famous after $24 \mathrm{~h}$ viewing its name) (Holden \& Vanhuele, 1999) all illustrate changes in consumer judgments following a single exposure to a stimuli without the attendant awareness of the prior exposure. Consequently, implicit tests, where participants are not told to remember events, but simply to perform some task, are increasingly recommended for measuring the impact of exposure (Krishnan \& Trappey, 1999). For implicit tests, memory is measured as a gain in performance (relative to an appropriate baseline), such as increased accuracy of identifying recently 
seen items relative to new items, or by a decrease in the amount of time necessary to identify recently seen items (for an extensive review, see Schacter, 1987).

It is argued here that if exposure to a product placement is best captured by implicit tests, then use of explicit tests (such as recognition or recall measures) would be uninformative and may even misrepresent the influence of the placement. Instead, it is believed that indirect tests of memory may be more appropriate. The present experiment tested that belief by directly comparing explicit and implicit memory measures for their responsiveness to product placement. The qualitative aspects of memory and memory errors were also investigated in an attempt to get a deeper understanding of the memory mechanisms involved in product placement.

\section{Review of Memory Measures and Predictions}

The distinction between implicit and explicit memory measures is particularly important for the present situation, because it has been shown that performance on an implicit memory measure can be uncorrelated or dissociated from performance on explicit memory tests (e.g., Graf \& Mandler, 1984; Holden \& Vanhuele, 1999; Jacoby \& Dallas, 1981; Shapiro, Maclnnis, \& Heckler, 1997), suggesting that different tests may be better at assessing different aspects of an exposure. As an illustration, Shapiro et al. (1997) report a study that utilized both an explicit and an implicit memory measure to document the effect of prior exposure on consumer behavior. Participants were required to read a magazine article delivered on a computer screen. The article was flanked by target ads designed to receive minimal reader attention. At the test phase participants were required to take an implicit memory test and an explicit memory test. For the implicit test they were provided with a buying scenario and asked to list eight products that would be included in their consideration set. No reference was made to the earlierviewed ad. The explicit test was a recognition test where they were asked to identify the target brand they had seen earlier from a list of four alternatives. Recognition rates for the experimental group were no different from those obtained from a control group that was not exposed to the ads, suggesting that exposure had no impact on the explicit test measure. The implicit test (i.e., the consideration set measure), however, revealed a different pattern of results. Participants were more likely to include the product featured in the ad in their consideration set compared to the control participants who had not viewed the ads. Similarly, Holden and Vanhuele (1999) have shown that fictitious brands may become instantly famous in the absence of recognition or conscious awareness, implying that brand reputation, which might affect brand choice, could be influenced without influencing recognition judgments (see also repetition-induced truth effect reported by Hawkins \& Hoch, 1992; Law et al., 1998). Taken 
together, these studies suggest that the effects of previous exposure on consumer judgment (such as brand reputation, validity of an advertising claim) and consideration-set formation can be reflected in an implicit test even when traditional explicit tests show null effects. This finding may explain why a majority of studies in the advertising literature report no direct relationship between advertising recall and sales effectiveness (Gibson, 1983), and in the present case, the inconclusive results observed in the product placement literature.

The primary goal of this research was to extend the literature on product placement by including different measures of memory-implicit and explicit-in a single study. In keeping with previous research (Krishnan \& Chakravarti, 1999; Krishnan \& Shapiro, 1996), the implicit measure employed in this study was a choice task that made no reference to the previous exposure episode. The explicit memory measures were recall and recognition. The inclusion of an implicit memory measure served an important purpose. Specifically, a weak (or insignificant) correlation between measures of explicit memory and implicit memory suggest dissociation between the two types of tests. Such demonstrations help delineate the conditions under which implicit memory tests are a more appropriate measure of product placement compared to explicit tests and visa versa. Under what conditions might implicit memory tests provide more appropriate measures for gauging the effectiveness of product placement?

Gupta and Lord (1998) have shown that products prominently placed (such as the Pepsi vending machine seen in Tom Hanks's living room in the film Big) are better remembered than products placed subtly (such as a Pepsi logo seen in the background of a scene). Reports from the popular press confirm that finding: Reese's Pieces candy sales increased by $65 \%$ in the 3 months following the release of the movie E. T. in which the candy was prominently displayed (Anonymous, 1989). However, some researchers studying the mere exposure effect find that those who have explicit memory of past-seen objects do not experience the same positive feelings as those who are unaware of that exposure (Ye \& Raaij, 1997). There is the possibility that placements could work by priming consumers to choose the products without the consumer being aware they had been influenced in that manner. Whether the placement works at an explicit or implicit level is important because factors such as depth of processing, which enhance explicit memory, have no effect on implicit memory (Schacter, 1987). Although prominence may influence explicit memory because of the potential for greater processing, such prominence may have little or no impact on implicit measures. Another goal for the present study was to examine whether the advantages of prominent placement on recall (an explicit test) are evident on other explicit tests (such as recognition) and on implicit measures such as choice. 
Modality of placement was also expected to have a different impact on explicit and implicit measures. Seen-only product placements are the most common, and audio visual presentations are the most expensive and hardest to accommodate (Gupta \& Lord, 1998). Product-placement consultants tell their clients that the best placement is one with a verbal mention, with the star actually using the product (Strauss, 1999). The consultants' recommendation is consistent with Paivio's (1986) dualcoding theory which posits that information may be coded in mental pictures and/or verbal codes. A seen-only placement is likely to be stored as a picture, whereas an auditory mention will be stored as a verbal code. Audio reinforced products have the benefit of being coded in both ways. The testing measures may differentially favor one type of coding over another; for instance, traditional recognition tests, which use brand names, may be biased toward auditory presentations because they are more consistent with the verbal coding. So far, empirical evidence on the modality of placements has been mixed. Some studies report that items presented with an audio reinforcement (i.e., audiovisual stimuli) lead to superior memory than visual placements (Sabherwal, Pokrywcynski, \& Griffin, 1994), particularly for subtle (instead of prominent) placements (Gupta \& Lord, 1998). By contrast, other studies have failed to replicate this finding (d'Astous \& Chartier, 1999; Lord \& Gupta, 1999). For example, d'Astous and Chartier (1999) compared seen-only placements with audiovisual placements and report that the mention of a brand by the principal actor had no significant effect on recognition memory, and in fact, was found to have a significantly negative impact on brand recall. We expect the use of implicit and explicit measures to add greater insight into the issue of modality.

\section{Exploring Qualitative Aspects of Memory}

In a typical recognition memory test participants are given a list of items consisting of new items and those seen earlier and asked to indicate whether or not they remember encountering the item previously. Such a test is limited in that it does not distinguish among responses based on accurate retrieval of the study episode (i.e., where the precise context or features of the previous encounter with the stimuli is recalled), responses based on guessing, or those based on general feelings of familiarity. Recently researchers have begun to dig deeper into the qualitative aspects of recognition; for instance, what the item looked like, whether recognition is based on feelings of familiarity or recollection of specific details, etc. Knowing these qualitative aspects of memory storage of product placements is important not only in designing effective testing methods, but also in manufacturing campaigns or instore displays that connect to the memory of the placement. Thus, in order to investigate the qualitative aspects of product placement on memory and pursue modality differences in exposure, the Memory 
Characteristics Questionnaire (MCQ) from Johnson, Foley, Suengas, and Raye (1988) was adapted for the recognition test, where participants indicate the context that led to their recognition (e.g., remembering hearing about the brand, recalling what it looked like, where it was located, etc.). An additional benefit of employing the MCQ scale was that it helps differentiate false "guesses" from true recollections, where fewer qualitative details ought to be associated with false alarms than true recollection. The MCQ scale also provides insight into whether the modality of the placement remains intact or if it moves to a more generic memory store.

\section{Experimental Investigation}

\section{Method}

Participants. The study took 45 minutes and involved 111 undergraduate students. ${ }^{1}$ Participants were told that the experiment involved rating a popular television program (Seinfeld). They were run in small groups and were randomly assigned to view one of two Seinfeld clips (Video A and B), lasting about $10 \mathrm{~min}$.

All participants indicated that they had watched and were aware of the Seinfeld show, with an average frequency of viewing of 5.2 on a 1-7-point scale where 1 = "very infrequently" and 7 = "very frequently." No significant differences were found between the two groups (Video $A=5.1$, Video $B=$ 5.2). Participants held very favorable feelings toward the Seinfeld show (Video $A=5.8$, Video $B=5.8$ ) on a 1-7-point scale, where 1 = "worst program" and 7 = "best program."

Materials. The two short Seinfeld clips were chosen as the experimental stimuli because they contained at least six unique products within a 10-min segment which fit the definitions of placement offered by Gupta and Lord (1998). See Table 1 for a description of the products placed. A seen-only condition involved seeing the product without hearing a mention of the brand name (e.g., seeing the Tide label in a garbage can). Heard-only conditions occurred when the product or brand name was mentioned in auditory form without the product itself being shown (e.g., the character Elaine referring to her airline seat companion as "vegetable lasagna"). In the audiovisual condition, products were both seen and heard (e.g., the character Kramer showing off his 3D art to Elaine's boss). The audiovisual placements also happened to be the more prominent placements in the segment and were used to test the centrality/ prominence hypothesis.

\footnotetext{
${ }^{1}$ Participants were run at two different universities. After no significant differences were found between the groups on the choice, recognition, or recall measures, or on Seinfeld viewing habits, the data were combined.
} 
Table 1. Product Placements in the Seinfeld Clips

\begin{tabular}{lccc}
\hline & Seen & Heard & Audiovisual \\
\hline Video 1 & & & \\
$\quad$ Poland Creek bottled water & $\checkmark$ & $\checkmark$ & $\checkmark$ \\
Coffee & & $\checkmark$ & \\
Muffins & $\checkmark$ & $\checkmark$ & $\checkmark$ \\
Eclair & $\checkmark$ & & \\
Tide laundry detergent & $\checkmark$ & & \\
3-D art & & $\checkmark$ & \\
Video 2 & $\checkmark$ & $\checkmark$ & \\
Vegetable lasagna & & & \\
Mott's apple juice & $\checkmark$ & & \\
Coconut & $\checkmark$ & $\checkmark$ & \\
Pepsi & & $\checkmark$ & \\
Pretzels & & \\
Gap clothing & & & \\
Play Now sports equipment & & & \\
\hline
\end{tabular}

Procedure

Participants were informed that the purpose of the study was to assess their evaluation of a popular network television show, Seinfeld. They were then assigned to watch either Video A or B. After having viewed the video, participants were asked to fill out an evaluation form rating their general impressions of the show, liking for the characters in the clip, and the frequency with which they watched Seinfeld.

After a short distraction, participants were administered the implicit memory measures. Participants were informed that they were participating in a different experiment for which they would be asked to shop for a friend who has just moved to a new apartment. They were given a list of items and brands preferred by the friend, and asked to check off the ones they would choose. No mention of the Seinfeld episode was made. The list included target items drawn from Videos A and B, and the critical effect of interest was the rate with which the participants chose the target products from the video episode seen earlier (Video A or B) relative to the baseline rate of choice by controls who saw a different episode (Video B or A). The difference between the exposure and control groups on the explicit and implicit tasks represents the facilitation due to the product placement.

After they had made the above choices, participants were asked to think back to the Seinfeld video clip and asked to recall as many products and brands from the episode as they could. Finally, participants completed a recognition test with the MCQ scale adapted by Johnson, Foley, Suengas, and Ray (1988). They were given a list of products - some of which were target products from the two videos, others were filler items - and asked to identify the ones they had seen in the video. For the 
items they recognized, participants had to indicate the bases for the judgment by checking off all relevant responses from a list of seven items that indicated whether they remembered (a) what was said about the product, (b) what was done with the product, (c) where the product was located, (d) what it looked like, (e) what they were feeling at the time of exposure, ( $f$ ) if their recognition was based on strong familiarity, and $(\mathrm{g})$ if their recognition was based on vague familiarity.

Results

Effects of Exposure on Implicit and Explicit Tasks. Table 2 presents the proportions for each of the three measures-recognition, recall, and choice-separately for each product as a function of whether the product had been present in the video (seen vs. control). Because each video contained six target products, products on Video A served as the control products for Video B and vice versa. Consequently, participants assigned to watch Video A provided baseline performance scores for participants assigned to watch Video B, and vice versa. Facilitation due to placement is defined as the difference between the exposure (seen) and control groups.

Table 2. Mean Proportions of Recognition, Recall, and Choice by Product

\begin{tabular}{|c|c|c|c|c|c|c|}
\hline \multirow[b]{3}{*}{ Products } & \multicolumn{4}{|c|}{ Explicit Measures } & \multirow{2}{*}{\multicolumn{2}{|c|}{$\begin{array}{c}\text { Implicit } \\
\text { Measure }\end{array}$}} \\
\hline & \multicolumn{2}{|c|}{ Recognition } & \multicolumn{2}{|c|}{ Recall } & & \\
\hline & Seen & Control & Seen & Control & Seen & Control \\
\hline \multicolumn{7}{|l|}{ Video A } \\
\hline Poland Creek bottled water & 85.71 & 5.36 & 60.71 & 0.00 & 30.36 & 25.00 \\
\hline Coffee & 66.07 & 48.21 & 7.14 & 0.00 & 32.14 & 39.29 \\
\hline Muffins & 21.43 & 8.93 & 0.00 & 1.79 & 39.29 & 23.21 \\
\hline Eclair & 91.07 & 14.29 & 50.00 & 0.00 & 7.14 & 5.36 \\
\hline Tide laundry detergent & 7.14 & 1.79 & 3.57 & 0.00 & 83.93 & 76.79 \\
\hline $3 \mathrm{D}$ art & 98.21 & 12.50 & 66.07 & 1.79 & 7.14 & 1.79 \\
\hline \multicolumn{7}{|l|}{ Video B } \\
\hline Vegetable lasagna & 41.82 & 12.73 & 3.64 & 1.82 & 34.55 & 32.73 \\
\hline Mott's apple juice & 74.55 & 5.45 & 54.55 & 0.00 & 40.00 & 27.27 \\
\hline Coconut & 74.55 & 3.64 & 21.82 & 0.00 & 20.00 & 14.55 \\
\hline Pepsi & 41.82 & 7.27 & 25.45 & 0.00 & 45.45 & 30.91 \\
\hline Pretzels & 23.64 & 12.73 & 3.64 & 1.82 & 36.36 & 20.00 \\
\hline Gap clothing & 49.09 & 9.09 & 12.73 & 1.82 & 60.00 & 61.82 \\
\hline Play Now sports equipment & 74.55 & 5.45 & 23.64 & 0.00 & 50.91 & 40.00 \\
\hline
\end{tabular}

Explicit Measures: Recognition and Recall Memory. From Table 2, it is clear that, as expected, exposure to a product resulted in better recognition memory for that product. A paired $t$ test performed on the recognition proportions showed a main effect of exposure, with overall recognition in the exposure group $(M=56.6)$ substantially higher than recognition in the control group $(M=11.1), t(12)=$ 
5.6, $p<.001$. Similar results showed increased memory in the exposure relative to the control group ( $M$ $=25.2$ vs. 0.7 , respectively) when recall performance was investigated; $t(12)=3.69, p<.01$.

Implicit Measure: Choice. Next, participants' choice proportions were examined for evidence of the impact of exposure. A comparison of choice proportions for the exposure and control conditions confirmed what is apparent in Table 2: There was an overall placement effect, with products placed in the episode being chosen more frequently $(M=36.8)$ than products not placed $(M=28.2), t(12)=5.0, p$ $<.001$. Thus, similar patterns of facilitation following placement were observed for all three measures employed in the study.

Relationship between Explicit and Implicit Memory Measures. To understand the relationship between the two explicit memory measures and the implicit choice measure, Pearson correlation coefficients were computed on the facilitation scores for recognition, recall, and choice, where facilitation was measured as the difference in choice (recognition, recall) rates between the experimental and control conditions. High correlations between participants' recognition and recall performance would signify that the two measures were tapping into the same type of memory. No correlation between the explicit and implicit measures would suggest dissociation between the two types of measures. Results showed that recognition facilitation was significantly correlated with recall facilitation (Pearson correlation coefficient of $0.88, p<.01$ ). Notably, the data revealed that facilitation scores in recognition memory (46.3\%) was greater than the effect observed for recall (24.9\%), suggesting that recognition task is a more pure measure of exposure effects than is recall. This difference was significant $-t(12)=5.5, p<.001-$ and this corresponds with past research in marketing where recognition is found to be a more sensitive measure of learning about products than recall (e.g., Singh \& Rothschild, 1983). More importantly, choice facilitation (implicit measure) was not significantly correlated with either explicit measure (with recall: $0.19, p>.1$; with recognition: $0.3, p>.1$ ).

Effect of Placement Centrality on Explicit and Implicit Memory Measures. Gupta and Lord (1998) have shown that placements prominently displayed in a movie have a greater impact on recall and recognition performance relative to when they are placed subtly. Similar results were also observed here, with prominent placement having positive benefits on each measure: recall, $F(1,12)=10.9, p<$ .01 ; recognition, $F(1,12)=13.1, p<.01)$. Table 3 gives the mean facilitation scores for each of the three dependent measures.

Most importantly, when the overall choice-facilitation data were broken down by centrality of placement, product placement did not produce a reliable effect on implicit choice facilitation $(F<1)$. This 
pattern of results bears resemblance to the dissociations reported in the cognitive psychology literature between implicit and explicit memory tests.

Effects of Modality of Placement on Explicit and Implicit Memory Measures. It was expected that modality of placement would have a different impact on explicit relative to implicit measures. The issue concerning placement modality is an important question for practitioners as well, because placement specialists frequently confront decisions about whether their product be seen, heard, or both. The question of whether there would be any trend as to what types of products were best remembered or more likely to be chosen was investigated.

Analysis on the recall scores revealed a significant main effect of modality, $F(2,331)=46.25, p<$ .001 , such that consistent with practitioners' beliefs and Paivio's dual coding theory, audiovisual placements were best recalled (49\% of participants who saw an audio/visual placement later freely recalled it vs. $20 \%$ recall for seen-only products and $9 \%$ recall of heard-only products). Recognition told a slightly different story about modality: audiovisual continued to be best remembered (with $87 \%$ correctly recognizing a placed item), but now heard-only products showed an advantage over seen-only products ( $54 \%$ for heard only and $37 \%$ for seen only). Modality was a significant factor at $F(2,331)=$ $45.39, p<.001$, and post hoc tests found all groups significantly different from one another. Notably, the implicit choice test revealed a completely different story: seen-only were most influential, where $47 \%$ of participants who had been exposed to a seen-only product later chose that product, compared to $34 \%$ for heard-only and $27 \%$ for audiovisual. The overall model was significant at $F(2,331)=15.54, p<.001$; post hoc tests revealed only the seen-only products to be significantly different from the heard-only and audiovisual (which were not found to be different from one another). Finally, although the recall and recognition (explicit) measures were highly correlated with one another $(r=0.5)$, neither correlated well with the choice (implicit) measure ( $r$ around 0 in both cases).

Table 3. Mean Percentage (and Standard Deviations) of Choice, Recall, and Recognition Facilitation Scores as a Function of Product Context (Central vs. Not Central)

\begin{tabular}{lcc}
\hline & \multicolumn{2}{c}{ Facilitation Scores } \\
\cline { 2 - 3 } & Central & Not Central \\
\hline \multirow{2}{*}{ Choice } & 5.8 & 9.02 \\
& $(3.7)$ & $(6.5)$ \\
Recall & 49.7 & 13.9 \\
& $(18.4)$ & $(17.8)$ \\
Recognition & 77.9 & 32.3 \\
& $(16.9)$ & $(24.2)$ \\
\hline
\end{tabular}


Qualitative Aspects of Placement Memories. The MCQ data were looked at to provide greater insight into the quality of the placed memories. Not surprisingly, individual subjects reported more MCQ items for the audiovisual products than either the heard-only or seen-only products; $M=2.71$ (of out a maximum of 7) for audiovisual; $M=1.39$ for heard-only products; $M=1.49$ for seen-only products. The overall model was significant at $F(2,220)=22.58, p<.001$, but post hoc tests found only the audiovisual products to be significantly different than the heard-only and seen-only products.

Whether the integrity of the sensory modality of the placement had been maintained in memory was also investigated. Table 4 gives the average frequencies of MCQ responses by the modality of product placement. As is evident from Table 4, participants' memory for the modality of placement was generally accurate. Analysis confirmed that seen-only products were most often recognized based on what they looked like: $49 \%$ of participants remembered what the seen-only product looked like compared to $21 \%$ of participants who chose that MCQ item for heard-only products, significantly different at $F(1,152) \_6.63, p<.1$. The heard-only products were more often remembered by what they sounded like than seen-only products, $0 \%$ vs. $20 \%$, significantly different at $F(1,152)=12.76, p<.001$.

Table 4. Mean Frequency Counts of MCQ Responses Across all Participants by Nature of Product Placement

\begin{tabular}{lccc}
\hline & \multicolumn{3}{c}{ Nature of Placement } \\
\cline { 2 - 4 } MCQ Response & Seen-Only & Heard-Only & Audiovisual \\
\hline Look & 39 & 18 & 134 \\
Did & 11 & 26 & 92 \\
Said & 20 & 92 & 127 \\
Location & 25 & 17 & 66 \\
Feeling & 5 & 9 & 34 \\
Strong familiarity & 14 & 19 & 69 \\
Vague familiarity & 12 & 3 & 4 \\
Other & 0 & & 0 \\
\hline
\end{tabular}

False Memories. In the recognition test, participants had to identify whether or not a product had appeared on the show. Overall, participants were good at identifying the correct items, with an average of 3.65 true and 1.26 false identifications per person. The MCQ scale provided insight on the quality of memories associated with true and false memories: Overall, there were more MCQ items associated with correct identifications, $M=1.9$, vs. false, $M=1.2$, significantly different at $t(238)=4.83$, $p<.0001$. Further analysis revealed that there were different types of MCQ items selected for true and false memories, with false ones eliciting more vague memories, $54 \%$, versus $22 \%$ for correct identifications, $t(87.5)=2.75, p<.001$. There was also much less sensory detail associated with false alarms, but when sensory information was wrongly remembered it was because participants 
remembered seeing the product (44 identifications), hearing the product (21), and action of product (25).

\section{Discussion}

The purpose of this study was to (a) determine the effectiveness of product placements within a TV program and (b) determine what type of testing methods might be most appropriate for measuring effectiveness. It was found that placements influenced the typically used explicit memory tests of recall and recognition and impacted the new implicit choice buying measure. Consistent with the memory literature, the implicit and explicit tests revealed different stories about placement effectiveness. Centrality of placement, which was influential on recall and recognition, showed no influence on choice. The effectiveness of modality of placement also differed depending on testing measure employed. Audiovisual placements were best remembered but least chosen. The recognition test appeared to have best helped memory for the heard-only products, perhaps because of the verbal nature of coding. The seen-only products were least recalled but most influential on choice, indicating that the facilitative effects of placements might be most influential if they are not consciously accessed. These results compel us to reevaluate the measures that are used as an index of product placement. Specifically, insofar as a choice measure is closer to how consumers behave in the marketplace than recognition or recall, these results suggest that the conventional measures of explicit memory may not capture (and may even mislead) how a placed brand may fare in the marketplace.

\section{Conclusions and Limitations}

This study affirms practitioner beliefs in the efficacy of product placement by replicating previous academic research suggesting product placements do have an impact. The effectiveness of placement, however, depends on how it is measured, with explicit memory tests revealing different results than implicit choice behavior. It appears the placements may be most effective when people are not particularly aware of their influence. This finding suggests that although cameo appearances by brand names in TV programs may accrue benefits for a marketer, it may be a dangerous trend from a public policy standpoint, blurring the distinction between advertising and programming. Because some groups, such as young children, may be more suggestible to placements, future policy research might consider setting standards regarding the manner in which products appear in TV shows.

It is likely product placement usage will increase as marketers continue to find nontraditional marketing vehicles to appeal to time-crunched and marketing-wary consumers. For those marketers, 
then, the question becomes one of judging placement effectiveness. One can measure if product placement increases top-of-mind awareness or brand accessibility, whether it facilitates the learning of brand name, if it improves a brand's chance of being considered for purchase, whether it influences product preferences, and so on. In this study, the interest was in contrasting the effectiveness of recall and recognition and determining whether choice was affected by prior exposure. Choice was not found to reliably correlate with recognition or recall performance. Not only was performance on one test uncorrelated with performance on the other, but independent variables were shown to differentially affect the two types of measures: The effects of centrality were always present in the explicit memory tasks, whereas the implicit choice task was not consistently affected for centrality, and a reversal occurred for modality. This suggests that past research supporting the rule of thumb regarding the superiority of placement prominence may be due to the usage of explicit tests and may not be informative of choice behavior.

Recent reports about the varying effectiveness of placements on the Seinfeld show bear on this issue: Kenny Roger's Roasters recently filed for bankruptcy, whereas placement of Junior Mints yielded large increases in brand awareness (Strauss, 1999). Of course, in these cases, many things, such as internal management issues, competitive action, etc., may be happening; the point is for managers to understand that there should be a match between the intent of a placement and the measures employed to gauge placement effectiveness. For example, if brand awareness is the primary motive, then impact of brand placement is best measured with an explicit (aided or unaided) recall test. On the other hand, if the marketers' main intent of the product placement is to increase a brand's likelihood of being chosen from an evoked or consideration set, implicit choice measures would best capture the placement's effectiveness. Interestingly, even within the present research's explicit measures of recall and recognition, different patterns of results occurred, which suggest that some of the inconsistency in past research efforts on product placements may be due to the testing mechanisms, for they ranged from recognition to free recall to cued recall tests.

The MCQ test revealed that the modality of placement was maintained in memory. This finding provides some guidance toward after-show marketing strategies to maximize placement benefits. For instance, for a product that was heard-only, a marketer could include point-of-purchase displays that contain an auditory cue to resurrect the memory trace of the placement in order to increase the likelihood of being chosen. Seen-only placements can be reinforced through imagery-based campaigns. Despite lower recall, as observed here, seen-only products might actually result in greater sales. In fact, the results could be underreporting this effect because the placed image is likely to connect more easily 
to the image of the product on the shelf than the grocery list task. Reinforcing how the product looked in the TV show could be a beneficial priming cue for creating positive affect toward the product, as researchers find that pictures or images can trigger stronger remembering than just words (Shacter, Koutstaal, Johnson, \& Gross, 1997). For products that are audiovisually placed, marketers can go beyond these sensory tactics into addressing the action of the TV program surrounding the product placement, because episodic memory appears to be organized around action-events (Anderson \& Conway, 1993). Recall alone of prominently placed products may not influence choice but perhaps a tie into the show may influence overall liking for the product (providing that such an association is positive).

As for limitations to this research, a potential confound in the current research should be noted, mainly that modality and centrality were confounded: Central products were those that had been both seen and heard. ${ }^{2}$ Future research should look at central (and noncentral) products that are seen and heard only in order to see whether it was the centrality that led to greater recall (and less choice) or the fact the product had appeared in both modalities. Furthermore, although care was taken to select a relatively old episode of Seinfeld, the chosen episodes may have seen by some of the participants. For instance, the influence on memory and choice may have carried over from an earlier exposure to the program and may not have been due to the single testing session. However, assuming the control groups may have had the same level of prior exposure, it can be concluded that the placement had some influence over and above just prior familiarity. In addition in his research, Karrh (1994) found that prior viewing made no difference. In his study, $63 \%$ of participants had previously seen the movie Raising Arizona and he found no significant differences in the cued recall scores and product evaluations between this group and the rest of the participants. A final objection could be raised that since no test of hypothesis guessing was conducted after choice, participants' choices may have been contaminated by their explicit recollection that the products had been seen in the just-viewed tape. In other words, the explicit measures may have influenced the implicit measures. Although this is a valid concern, the experiment was designed so that the implicit task appeared before the explicit memory measures in order to avoid participants being consciously aware that product placements were being investigated. In addition, two pieces of evidence suggest that this may not be a serious concern. First, correlations between the explicit and implicit measures were weak and insignificant, suggesting that one measure was not informative of the other (the assumption is that in this scenario, explicit memory, when available, would oppose the influence of implicit memory). Second, dissociations were observed where the audiovisual placements were best remembered but least chosen. Had the participants been actively

\footnotetext{
${ }^{2}$ We thank a reviewer for raising this issue.
} 
thinking that they should pick the placed product, more of the better-recalled products should have been chosen. Nonetheless, the issue of contamination is an important one from the methodological perspective and should be explored in future research. From a practical perspective, it is notable that placement had an influence on choice despite the possibility of this contamination.

The present study used real-world TV segments to judge placement effectiveness, which helps create external validity but detracts from internal control. One advantage in the use of the Seinfeld episodes over previous studies is that the segments contained multiple types of placements. Future research should exert more control over the size and duration of the placements. Controlling for modality between products (e.g., see Pepsi in one episode and hear it in another) will also help gain greater insight on how sensory modality influences choice behavior. Finally, this research did not investigate the issue of how a product is portrayed in the placement-in a negative or a positive light. ${ }^{3}$ This is an important issue for two reasons. First, given that not all placements are portrayed favorably and that marketers often have little control over the context in which the product is embedded, it will be important to know whether and how placement contexts affect placement effectiveness. Second, as Karrh (1995) notes, "portraying the product or service in a favorable light" is a critical concern of practitioners. Therefore, future research in which the effect of negative and positive brand depictions are assessed would help verify and extend the present research's findings.

\section{References}

Anderson, S. J., \& Conway, M. A. (1993). Investigating the structure of autobiographical memories. Journal of Experimental Psychology: Learning, Memory, and Cognition, 19, 1178-1196.

Anonymous. (1989, January 2). Plugging away in Hollywood. Time, p. 103.

Babin, L. A., \& Carder, S. T. (in press). Advertising via the box office: Is product placement effective? Journal of Promotion Management.

Babin, L. A., \& Carder, S. T. (1996). Viewers' recognition of brands placed within a film. International Journal of Advertising, 15, 140-151.

Buss, D. D. (1998). Making your mark in movies and TV. Nation's Business, 86(12), 28-32.

d'Astous, A., \& Chartier, F. (1999). How should we plug our brand? A study of factors affecting consumer evaluations and memory of product placements in movies. In Y. Evrard, W. D. Hoyer, \& A.

\footnotetext{
${ }^{3}$ We thank a reviewer for this suggestion.
} 
Strazzieri (Eds.), Proceedings of the Third International Research Seminar on Marketing Communications and Consumer Behaviour (pp. 104-117). IAE d'Aix-en-Provence.

Gibson, L. D. (1983). If the question is copy testing, the answer is..."Not recall." Journal of Advertising Research, 23(1), 39-46.

Graf, P., \& Mandler, G. (1984). Activation makes words more accessible, but not necessarily more retrievable. Journal of Verbal Learning and Verbal Behavior, 23, 553-568.

Gupta, P. B., \& Lord, K. R. (1998). Product placement in movies: the effect of prominence and mode on recall. Journal of Current Issues and Research in Advertising, 20(1), 47-59.

Hawkins, S. A., \& Hoch, S. J. (1992). Low-involvement learning: Memory without evaluation. Journal of Consumer Research, 19, 212-225.

Holden, S. J. S., \& Vanhuele, M. (1999). Know the name, forget the exposure: Brand familiarity versus memory of exposure context. Psychology and Marketing, 16, 479-496.

Jacoby, L. L., \& Dallas, M. (1981). On the relationship between autobiographical memory and perceptual learning. Journal of Experimental Psychology: General, 110, 306-340.

Janiszewski, C. (1993). Preattentive mere exposure effects. Journal of Consumer Research, 20, 376-392.

Johnson, M. K., Foley, M. A., Suengas, A. G., \& Raye, C. L. (1988). Phenomenological characteristics of memories for perceived and imagined autobiographical events. Journal of Experimental Psychology: General, 117, 371- 376.

Karrh, J. A. (1994). Effects of brand placements in motion pictures. Paper presented at the 1994 Conference of the American Academy of Advertising, Athens, GA.

Karrh, J. A. (1995). Brand placements in feature films: The practitioners' view. Paper presented at the 1995 Conference of the American Academy of Advertising, Waco, TX.

Krishnan, H. S., \& Chakravarti, D. (1999). Memory measures for pretesting advertisements: An integrative conceptual framework and a diagnostic template. Journal of Consumer Psychology, 8(1), 1-37.

Krishnan, H. S., \& Shapiro, S. (1996). Comparing implicit and explicit memory for brand names from advertisements. Journal of Experimental Psychology: Applied, 2, 147-163.

Krishnan, H. S., \& Trappey, C. V. (1999). Nonconscious memory processes in marketing: A historical perspective and future directions. Psychology and Marketing, 16, 451-457.

Law, S., Hawkins, S. A., \& Craik, F. I. M. (1998). Repetition-induced belief in the elderly: Rehabilitating age-related memory deficits. Journal of Consumer Research, 25, 95-107. 
Lord, K. R., \& Gupta, P. B. (1999). Response to brand placement: The effects of context and placement characteristics. Working paper, Mercer University.

Paivio, A. (1986). Mental representativeness: A dual coding approach. New York: Oxford University Press.

Sabherwal, S., Pokrywcynski, J., \& Griffin, R. (1994). Brand recall for product placements in motion pictures: A memory-based perspective. Conference proceedings of AEJMC 1994 Ad DivisionResearch Track. Atlanta, GA.

Schacter, D. L. (1987). Implicit memory: History and current status. Journal of Experimental Psychology: Learning, Memory, and Cognition, 13, 501-518.

Schacter, D. L., Koutstaal, W., Johnson, M. K., \& Gross, M. S. (1997). False recollection induced by photographs: A comparison of older and younger adults. Psychology and Aging, 12, 203-215.

Shapiro, S., MacInnis, D., \& Heckler, S. (1997). The effects of incidental ad exposure on the formation of consideration sets. Journal of Consumer Research, 24, 94-104.

Singh, S. N., \& Rothschild, M. L. (1983). Recognition as a measure of learning from television commercials. Journal of Marketing Research, 20, 235-248.

Strauss, G. (1999, February 24). Seinfeld gives mixed signs. USA Today, p. 1 B.

Vollmers, S., \& Mizerski, R. (1994). A review and investigation into the effectiveness of product placements in films. Conference proceedings of the 1994 Conference of the American Academy of Advertising (pp. 97-102). Athens, GA: American Academy of Advertising.

Wells, S. (1996, June 4). TV's subtle sell. USA Today, p. $1 B$.

Ye, G. W., \& Raaij, W. F. (1997). What inhibits the mere-exposure effect: Recollection or familiarity? Journal of Economic Psychology, 18, 629-648. 